\title{
Chemiluminescence Induced by Phagocytosis of Escherichia coli by Polymorphonuclear Leucocytes
}

\author{
By ALI FAZELI AND LEE RICHARDS* \\ Department of Microbiology and Isotope Unit, Queen Elizabeth College, Campden Hill Road, \\ Kensington, London W8 $7 A H, U K$
}

(Received 12 September 1983; revised 8 June 1984)

\begin{abstract}
Chemiluminescence emitted by phagocytosing human polymorphonuclear leucocytes stimulated by Escherichia coli was measured using a liquid scintillation counter equipped with a multichannel analyser. In the presence of the amplifying agent luminol, light emission can be divided into two channels, one of which ('high energy') appears to correlate directly with phagocytic activity of the PMNL, and the other ('low energy') with the background luminol dioxygenation by the cells. Measuring in the 'high energy' window also eliminates the normal 'out of coincidence' background. The method is applicable to measuring opsonizing capacity of different sera, and responds to PMNL number, age, composition of assay medium and the integrity of the stimulating bacteria. Other bacterial strains produce a similar response, as does the artificial stimulator zymosan. Low temperature and anaerobiosis, which inhibit phagocytic killing, also suppress light emission.
\end{abstract}

\section{INTRODUCTION}

Generation of light by phagocytosing polymorphonuclear leucocytes (PMNL), a phenomenon related to the respiratory burst induced by phagocyte-bacteria contact, was first described by Allen et al. (1972). The chemiluminescence produced can be measured in a liquid scintillation spectrometer, or in a fluorimeter with the actinic illumination blanked off (Van Dyke et al., 1977).

The close correspondence observed between the chemiluminescence emitted and the microbicidal activity of the phagocyte (Grebner et al., 1977; Matthay et al., 1981) has enabled measurement of light emitted to be used as a sensitive tool for the study of serum opsonic activity (Easmon et al., 1980), phagocyte potency (Mills et al., 1980), and diagnosis of bacterial infection (Barbour et al., 1980) as well as to distinguish between bacterial and viral infections (McCarthy et al., 1980). One difficulty with this assay procedure compared with conventional clinical assays is the requirement for a large number of phagocytic cells and expensive instrumentation. The speed of the assay, however, and its precision, can be far superior to that of viable count based techniques.

The number of phagocytic cells required can be greatly decreased by amplifying the light emission by addition of compounds such as luminol (5-amino-2,3-dihydro-1,4-phthalazinedione) (Allen \& Loose, 1976) or luciferin or lucigenin (Henry \& Michelson, 1977; Williams \& Cole, 1981). The greater light output also permits measurement with small and inexpensive light detectors, rather than scintillation counters. Comparisons of chemiluminescence in the presence or absence of luminol indicate that both are reflecting the same metabolic events, though with different sensitivity (Wilson et al., 1978). Indeed, measurement with luminol present was more highly correlated with the carrier state of chronic granulomatous disease than other methods, including inherent chemiluminescence.

Abbreviations: HBSS, Hanks' balanced salts solution; PMNL, polymorphonuclear leucocytes. 
The chemiluminescence produced is correlated with a number of factors such as number, type and age of phagocytes used (Stevens \& Young, 1977), bacteria :phagocyte ratios (Allen, 1977), opsonic capacity of serum (Hemming et al., 1976), nature of test bacterium (Kossack et al., 1981), and availability of oxygen (Allen, 1979).

The method used to prepare the PMNL, particularly the method used to lyse residual erythrocytes, influences the assay results, as residual erythrocytes substantially depress the chemiluminescence obtained (Andersen \& Brendzel, 1978). Assay temperature and cell aggregation during the measurement also exert an influence (Andersen \& Amirault, 1979; Easmon et al., 1980), thus necessitating a precisely standardized and reproducible assay procedure.

The number of pulses detected in a scintillation counter is usually measured, irrespective of the 'pulse height' or energy, in a 'wide open' window. In this paper, chemiluminescence emitted by phagocytes ingesting Escherichia coli was assayed and subjected to multichannel pulse height analysis. The effect of various assay conditions on the energy distribution was determined.

\section{METHODS}

Bacteria and polymorphonuclear leucocytes. The methods used for the preparation of polymorphonuclear leucocyte suspensions in Hanks' balanced salts solution (HBSS), and of suspensions of bacteria opsonized with autologous serum were as previously described (Fazeli \& Richards, 1983).

Measurement of phagocytosis-dependent chemiluminescence. Light emission induced by phagocytosis of Escherichia coli by human PMNL was measured at ambient temperature in a model NE8316 CAMAC liquid scintillation counter (Nuclear Enterprises, Edinburgh, UK), equipped with EMI 9635 QB photomultiplier tubes, and linked to and controlled by a PDP $11 / 05$ computer (Digital Equipment, Galway, Ireland). Signal amplification was pseudologarithmic, and pulse height analysis was carried out by an internal multichannel analyser which divided the energy spectrum into 256 equal divisions (channels) spanning the energy range 0 $350 \mathrm{keV}$. Two counting vials were used in each experiment, one a control lacking some ingredient of the assay, and they were counted alternately for $40 \mathrm{~s}$ in each $2 \mathrm{~min}$ period for a total of $180 \mathrm{~min}$. Data for each vial were expressed as c.p.m.

In some experiments, where light emission at $4^{\circ} \mathrm{C}$ was investigated, a Packard Tri-Carb model 3003 liquid scintillation counter was used. All manipulations were carried out under red light (Ilford F904 safelight) using prevously dark-adapted polystyrene counting vials.

Each vial, containing 2 to $3 \times 10^{6} \mathrm{PMNL} \mathrm{ml}^{-1}$ in $2 \mathrm{ml} \mathrm{HBSS}$, at $\mathrm{pH} 7 \cdot 2$, and $10^{-5} \mathrm{M}$-luminol, was counted for 5 min continuously to achieve a stable background, then phagocytosis was initiated by addition of $1 \mathrm{ml}$ HBSS containing 2 to $3 \times 10^{7} \mathrm{c}$.f.u. of opsonized bacteria to one vial, and a similar volume of HBSS alone to the other.

Measurement of chemiluminescence under anaerobic conditions. HBSS was bubbled with oxygen-free nitrogen for $12 \mathrm{~h}$ before use, and PMNL and bacterial pellets were resuspended separately in this using an anaerobic cabinet (National Applicance Co., Portland, Oregon, USA). PMNL suspension $(1 \mathrm{ml}$ ) and the required amount of luminol were placed in scintillation vials while $1 \mathrm{ml}$ of bacterial suspension or the same volume of HBSS (as control) was placed in a small polypropylene sample tube inside the scintillation vials. After $10 \mathrm{~min}$ background counting, bacteria and PMNL were mixed by repeated inversion of the vials, and recounted in the Nuclear Enterprises counter for $120 \mathrm{~min}$.

Cell-free luminol activation. Cell-free chemiluminescence was generated by the method described by Mason et al. (1978). All reagents were freshly made up in deionized water. Two vials were used, each containing luminol stock solution, $0.05 \mathrm{M}$-sodium hydroxide and deionized water in the ratio $5: 6: 5$, by vol., and $0.2 \mathrm{ml} 10^{-3} \mathrm{M}$-ferric chloride. After $10 \mathrm{~min}$ background counting, $0.2 \mathrm{ml} 5 \times 10^{-2} \mathrm{M}$-sodium perborate was added to one vial, and the same volume of water to the other as control. Other reagents were added as necessary, and counting continued.

Cell disruption. Bacteria were ultransonically disrupted using an MSE $150 \mathrm{~W}$ sonicator with a titanium microprobe ( $3 \mathrm{~mm}$ diameter). Samples were cooled in iced water during treatment.

Reagents. Luminol (Sigma) stock solution was prepared by dissolving the solid in dimethyl sulphoxide to a concentration of $2 \mathrm{mg} \mathrm{ml}^{-1}$. This was stable for more than one month at ambient temperature. Other reagents were from routine suppliers, and were of the highest purity obtainable.

\section{RESULTS}

\section{Phagocytosis-induced chemiluminescence in the presence of luminol}

With the scintillation counter operated 'in coincidence', multichannel pulse height analysis of luminol-amplified chemiluminescence showed a pronounced difference between the energy 

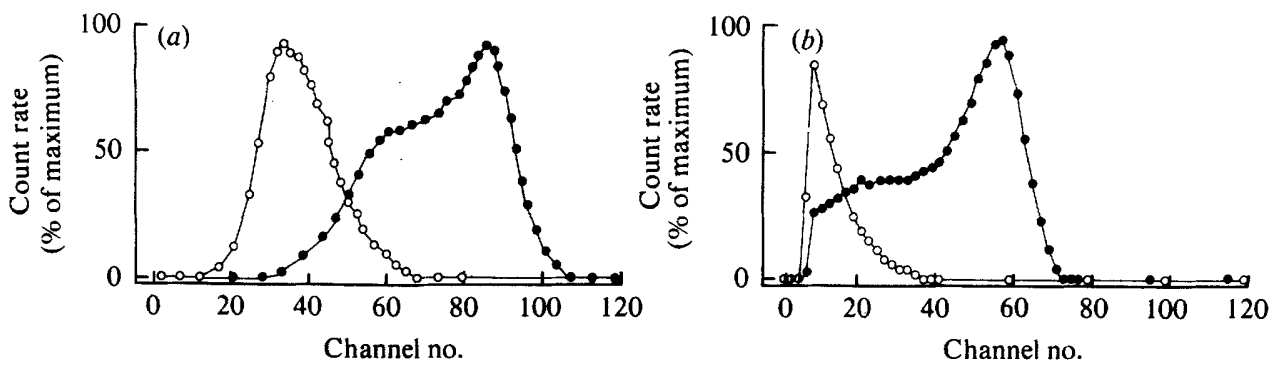

Fig. 1. Pulse height spectrum of phagocytosis-induced chemiluminescence by human PMNL, (a) using gated coincidence and $(b)$ in 'out of coincidence' mode. 4 to $6 \times 10^{6} \mathrm{PMNL}$ in HBSS, at pH 7.2, and $10^{-5} \mathrm{M}$-luminol in a volume of $2 \mathrm{ml}$ were equilibrated in dark-adapted vials. Background counts were made and $1 \mathrm{ml} \mathrm{HBSS}(O)$, or $1 \mathrm{ml}$ opsonized $E$. coli suspension containing 2 to $3 \times 10^{7}$ cells $(O)$ added. The figure shows the spectrum $30 \mathrm{~min}$ after initiation of phagocytosis. The $100 \%$ value corresponds to $2^{16}$ counts accumulated in the modal channel.
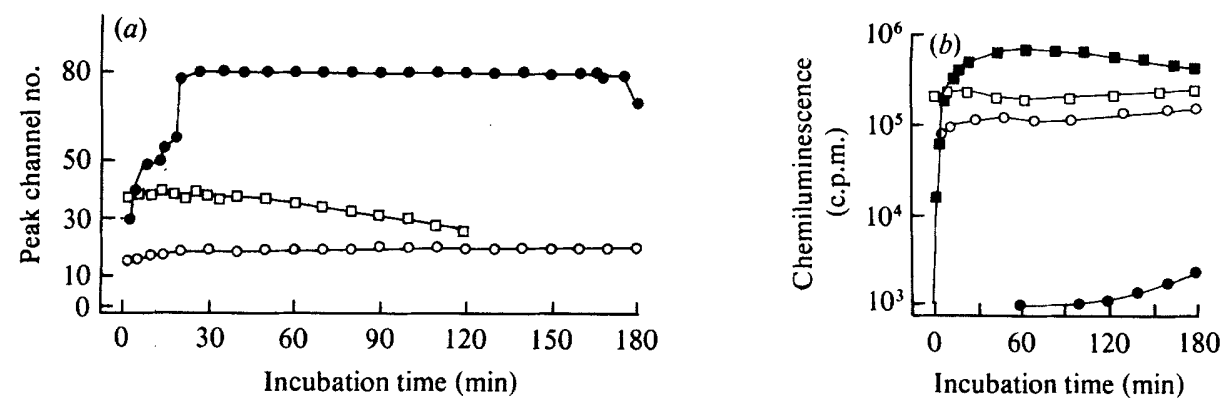

Fig. 2. Time course of phagocytosis-induced chemiluminescence. (a) Modal channel number determined from pulse height analysis of light emission measured in gated coincidence under experimental conditions identical with those for Fig. 1. O, Resting PMNL; $\square$, PMNL challenged with non-oposonized $E$. coli; , PMNL challenged with opsonized $E$. coli. (b) Count rate in 'high energy' channel (filled symbols) and 'low energy' channel (open symbols). $\bigcirc$, Resting PMNL; $\square, \square$, PMNL challenged with opsonized $E$. coli.

spectra produced by phagocytosing and resting PMNL, with the former having the higher energy (Fig. $1 a$ ). This led to the chemiluminescence energy spectrum being integrated over two regions: channels 1-50 (the 'low energy' window) and channels 51-120 (the 'high energy' window). The emission by resting PMNL exhibited a symmetrical spectrum, whilst that from phagocytosing PMNL showed a characteristic shape with a 'shoulder' to the peak. The reason for the presence of this shoulder is not known.

When the counter was operated 'out of coincidence', all events detected by the photomultipliers were included, and the energy spectra observed with resting or phagocytosing PMNL are shown in Fig. 1(b). In this case, the count rate in both channels was larger, as would be expected, and the largest count was in the 'low energy' window. This presumably reflects both the inclusion of 'low energy' photomultiplier noise (which is the reason for the use of gated coincidence in scintillation counting), and the addition of some chemiluminescent counts which were previously excluded as they failed to undergo chance coincidence. The shape of the spectrum seen with resting cells was distorted by the addition of this extra 'very low energy' count. That produced by phagocytosing PMNL was essentially unchanged, but the whole spectrum appeared at lower energy, with a peak around channel 60 rather than the normal channel 80 as typically shown in Fig. $1(a)$.

The differences in the pulse height spectrum could also be monitored by the shift in the modal channel of emission, as shown in Fig. 2(a). The modal channel of the emission was higher with active PMNL than with resting PMNL, and was also higher when opsonized E. coli were used as substrate rather than non-opsonized ones. 
The rate of chemiluminescence, as measured in the 'high energy' window, typically reached a maximum 30-60 min after addition of bacteria to the PMNL, and remained constant for 30$60 \mathrm{~min}$ thereafter, followed by a slow decline up to about $180 \mathrm{~min}$, and thereafter a rapid decline (Fig. $2 b$ ). The 'low energy' count rate reached a plateau almost immediately after addition of bacteria to PMNL, and remained constant thereafter, even when fresh bacteria were added into the mixture.

The fall in 'high energy' count rate seen after about $90 \mathrm{~min}$, and the more rapid fall after $180 \mathrm{~min}$ could not be attributed to exhaustion of the bacterial phagocytosis 'substrate', since the addition of fresh bacteria at $210 \mathrm{~min}$ did not halt this decline. To test whether it could have been due to inactivation or consumption or luminol, a phagocytosis mixture which had been incubated for $18 \mathrm{~h}$ was centrifuged at $4000 \mathrm{~g}$ for $15 \mathrm{~min}$ to remove the cells, and the supernate was reincubated with fresh PMNL and opsonized bacteria. There was no significant difference between the chemiluminescence measured (using either energy window) with either fresh luminol, pre-used luminol, or luminol which had been incubated at ambient temperature in HBSS for $18 \mathrm{~h}$. Thus the decline was attributed to a decrease in phagocyte activity.

\section{Effect of medium constituents and assay conditions on phagocytosis-induced chemiluminescence}

Preliminary experiments showed that supplementation of HBSS with $0.1 \%(w / v)$ gelatin increased the chemiluminescence observed in the 'high energy' window by $100 \%$ at first, and by $41 \%$ after $30 \mathrm{~min}$ of phagocytosis, and consequently gelatin was routinely added to all assays.

Comparison of chemiluminescence emitted at $4{ }^{\circ} \mathrm{C}$ and at $24^{\circ} \mathrm{C}$ showed essentially no chemiluminescence in the first $10 \mathrm{~min}$ at $4^{\circ} \mathrm{C}$, whilst at $24^{\circ} \mathrm{C}$ there were about $9 \times 10^{5}$ c.p.m. In the purely chemical luminol oxidation system, there was no significant difference between emission measured at $4^{\circ} \mathrm{C}$ and $24^{\circ} \mathrm{C}\left(10^{6}\right.$ c.p.m. $)$.

The emission rate in the 'high energy' window was also linearly related to the bacterial c.f.u. : PMNL ratio, while that in the 'low energy' window was essentially independent of this ratio. The standard assay method used a 10:1 ratio of bacteria to PMNL, with the PMNL numbers held at 2 to $3 \times 10^{6} \mathrm{ml}^{-1}$.

The effect of post-venipuncture age of the PMNL on chemiluminescence elicited by phagocytosis was investigated using cells which had been stored in HBSS at room temperature for different lengths of time. There was no significant change during the first $6 \mathrm{~h}$ of storage, but a steady decrease thereafter up to $30 \mathrm{~h}$ in the 'high energy' window, with no differences apparent in the 'low energy' window (Table 1).

Elimination of oxygen from the phagocytosis mixture reduced light emission in both channels (by $88 \%$ in the 'low energy' and $99.9 \%$ in the 'high energy' window), although the absence of oxygen did not decrease light emission in the cell-free luminol activation system.

\section{Opsonin requirement for chemiluminescence response}

The count rates produced when PMNL were stimulated with human serum-opsonized, human plasma-opsonized, calf serum-opsonized, and non-opsonized bacteria were compared. Although a light emission significantly above background was detected with non-opsonized bacteria, it was very much less than with bacteria opsonized by any of the above methods. There was no significant difference between human plasma or serum opsonization, at least after the first few minutes of incubation, while calf serum was a much less effective opsonizing agent (Table 2). When non-opsonized bacteria were used, the peak of 'high energy' emission occurred at $60 \mathrm{~min}$, then it stayed constant for $90 \mathrm{~min}$. It declined thereafter to less than 1000 c.p.m. by $4 \mathrm{~h}$, at which time the 'low energy' count rate was still more than $10^{5}$ c.p.m. (Fig. 3). Fresh plasma-opsonized E. coli $\left(4 \times 10^{7}\right.$ c.f.u. $)$ were added at time $270 \mathrm{~min}$, and almost immediately the 'high energy' count rate rose, reaching a maximum of $6 \times 10^{5}$ by $30 \mathrm{~min}$, although it then declined sharply. The 'low energy' count rate rose slightly after the addition of the opsonized bacteria, and then continued to decline. The emission with non-opsonized bacteria was substantially less than with opsonized ones, and it also had a different energy spectrum, with a peak at lower energy (channel 40, see Fig. $2 a$ ). It may have been recorded as unduly low since the energy window was not the optimum. 


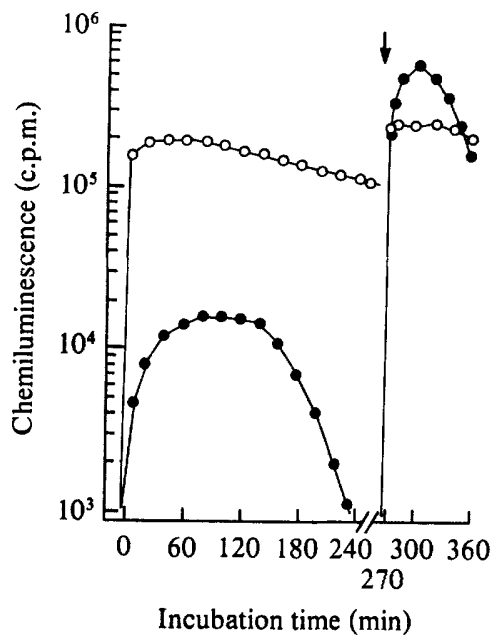

Fig. 3. Effect of adding fresh opsonized $E$. coli to PMNL which were phagocytosing non-opsonized $E$. coli. ' 'High energy' window count rate; $O$, 'low energy' window count rate when $2 \times 10^{6}$ PMNL ml ${ }^{-1}$ were challenged with $2 \times 10^{7}$ non-opsonized $E$. coli $\mathrm{ml}^{-1}$ in the presence of $10^{-5} \mathrm{M}$ luminol in HBSS. At 270 min an equal number of opsonized bacteria were added.

Table 1. Variation of chemiluminescent response with post-venipuncture age of $P M N L$

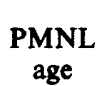

(h)

4
24
30

\begin{tabular}{|c|c|c|c|}
\hline \multicolumn{2}{|c|}{$10 \min \dagger$} & \multicolumn{2}{|c|}{$30 \min \dagger$} \\
\hline HE & LE & HE & LE \\
\hline $\begin{array}{l}1.2 \pm 0.3 \\
0.87 \pm 0.1 \\
0.72 \pm 0.18\end{array}$ & $\begin{array}{l}1.8 \pm 0.05 \\
1.8 \pm 0.06 \\
1.7 \pm 0.06\end{array}$ & $\begin{array}{l}9.9 \pm 0.9 \\
7.9 \pm 0.7 \\
7.2 \pm 1\end{array}$ & $\begin{array}{l}6.6 \pm 1 \\
6.6 \pm 0.9 \\
6.5 \pm 1.2\end{array}$ \\
\hline
\end{tabular}

* Light emission by $4 \times 10^{6} \mathrm{PMNL}$ and $4 \times 10^{7} \mathrm{E}$. coli in $2 \mathrm{ml} \mathrm{HBSS}$, containing $10^{-5} \mathrm{M}$-luminol. The PMNL (which were all collected at the same time from a single donor) were stored in HBSS containing $0.1 \%$ gelatin at room temperature for different periods before use.

$\dagger$ Results shown are the total light emission $\left(10^{-6} \times\right.$ c.p.m.) up to the stated time of incubation (mean of five assays $\pm \mathrm{SD}$ ). $\mathrm{HE}$, counts in 'high energy' window; LE, counts in 'low energy' window.

Table 2. Variation in chemiluminescent response with method of opsonization of $E$. coli

\begin{tabular}{lcc}
$\begin{array}{c}\text { Opsonin } \\
\text { source }\end{array}$ & $\begin{array}{c}10^{-6} \times \text { Total 'high energy' counts* } \\
\text { in mixture after incubation for: }\end{array}$ \\
\cline { 2 - 3 } $\begin{array}{c}\text { Human serum } \\
\text { Human plasma }\end{array}$ & $2.4 \pm 0.37$ & $30 \mathrm{~min} \dagger$ \\
Calf serum & $3.7 \pm 0.19$ & $6.1 \pm 1.6$ \\
& $0.11 \pm 0.04$ & $0.37 \pm 0.29$ \\
\end{tabular}

\footnotetext{
* Integrated light emission, measured up to stated time of incubation, when $4 \times 10^{6} \mathrm{PMNL}$ in $2 \mathrm{ml}$ HBSS with $10^{-5} \mathrm{M}$-luminol were challenged with $4 \times 10^{7} \mathrm{E}$. coli opsonized by $30 \mathrm{~min}$ incubation at $37^{\circ} \mathrm{C}$ in the preparations listed, followed by washing and resuspension in HBSS. 'High energy' window counts only shown; all are $10^{-6} \times$ c.p.m.
}

$\dagger$ Figures represent mean of four assays \pm SD.

\section{Chemiluminescence response of PMNL to different stimulating particles}

In an experiment in which the bacterial and PMNL numbers were kept constant (and using a single donor as source of PMNL), the magnitude of the chemiluminescence produced by different kinds of bacteria was investigated. Fig. 4 shows the results relative to the emission 


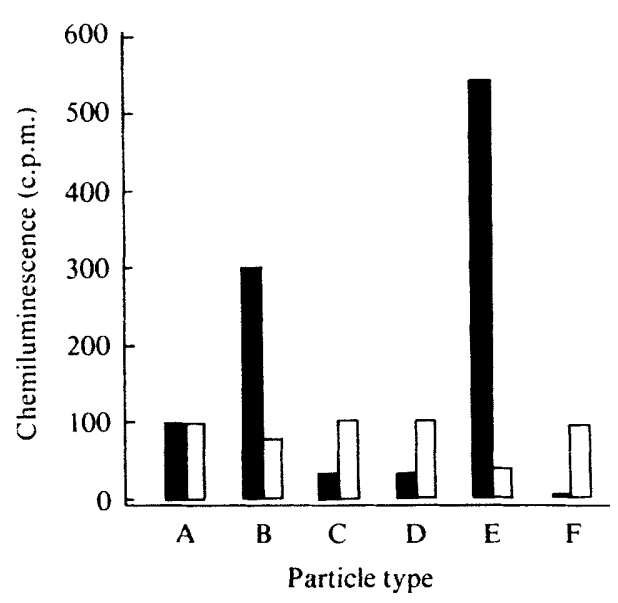

Fig. 4. Relative amounts of chemiluminescence produced by $2 \times 10^{6}$ PMNL from a constant source, when challenged by $2 \times 10^{7}$ cells of different bacterial strains, all opsonized similarly, or by $2 \mathrm{mg}$ zymosan $\mathrm{ml}^{-1}$. Open columns represent integrated light emission up to $30 \mathrm{~min}$ in the 'low energy' window, filled columns the 'high energy' window. All are expressed as a percentage of 'high energy' emission with $E$. coli $0-86\left(8.19 \times 10^{6} \pm 0.82\right.$ c.p.m. $)$, and are the means of from four to six experiments. A, E. coli O-86; B, Staphylococcus aureus C-18; C, E. coli B-3; D, E. coli B-10; E, zymosan; $F$, non-phagocytosing PMNL. Values shown are $\pm 6.4 \%$.

produced using $E$. coli O-86. Staphylococcus aureus C-18 produced a threefold higher response, and the artificial stimulator zymosan at $2 \mathrm{mg} \mathrm{ml}^{-1}$ gave even higher light emission. Strains B-3 and $\mathrm{B}-10$ of $E$. coli gave a reduced light output. When the experiment was done using $E$. coli O-86 rendered non-viable by either heat, streptomycin or sonication treatment, chemiluminescence in the 'high energy' channel was greatly decreased or even eliminated altogether whilst that in the 'low energy' channel was only slightly decreased.

\section{DISCUSSION}

The phenomenon of phagocytosis-induced chemiluminescence has frequently been used to study both humoral dysfunctions (Williams et al., 1980) and cellular dysfunctions (Mills et al., 1980; Rosen \& Klebanoff, 1976). The involvement of the oxidative respiratory burst in the mechanism of the response has been shown by Allen $(1975 a, b)$. Previous work, however, has customarily measured the light emission in an 'out of coincidence' scintillation counter with a 'wide open' window, or in a fluorimeter, without doing any pulse height analysis on the signals from the detector. The present work showed that it was possible to characterize the emission using automated pulse height analysis, and an 'in coincidence' scintillation counter. Unlike the situation in the normal use of a gated coincidence counter for measurement of $\beta$ radioactivity, the chemiluminescent emission accompanying phagocytosis represents single photon events, which will only pass through gated coincidence due to chance coincidences at high emission rates, and the greater the number that do so, the larger the apparent 'energy' as measured by the pulse height spectrum. Nonetheless, in practice it appears that the method can be successfully applied to the measurement of phagocytosis, and that two 'energy windows' may be distinguished. The counts measured in the 'high energy' window appeared to correlate directly with the phagocytic activity of the PMNL, and responded similarly to factors influencing this activity (such as bacterial and PMNL numbers, assay medium and so on) as do 'open' window measurements in the absence of gated coincidence. Further, the 'low energy' window appeared 
to contain activity which may be taken as the 'background' luminol oxidizing activity of PMNL in the absence of phagocytosis, since it represents a count rate above the background, produced by PMNL, which does not correlate with phagocytic ability. Presumably it corresponds to luminol oxidation brought about by haem compounds and oxidizing species produced by the resting phagocyte. Further, resolution of the activity into two channels enabled measurements to be made (in the 'high energy' window) free of the usual very high background count which accompanies such experiments done with a counter with the coincidence gate turned off, and consequently gave greater sensitivity.

It is clear from these results that a rise in 'high energy' count rate occurred when PMNL were challenged with bacteria or zymosan, that it reached a peak which was sustained for a few hours, and that it then gradually declined presumably due to a decline in the phagocytic capacity of the PMNL, since the addition of fresh bacteria at this point did not halt the decline, which was not due to exhaustion of the luminol.

Addition of fresh, opsonized bacteria to PMNL after $4 \mathrm{~h}$ incubation with non-opsonized bacteria gave an increase in light emission to a level characteristic of the ingestion of normal opsonized cells. The spectral difference seen between chemiluminescence elicited by opsonized and non-opsonized bacteria suggested that the emission could be resolved into more than the two broad windows used here, which may in turn enable the processes leading to uptake of opsonized and/or non-opsonized bacteria to be measured independently.

Some substrates are more potent stimulators of post-phagocytic metabolism than others (Mandell, 1971) and the results showed that the extent to which different particles stimulate chemiluminescence measured in the 'high energy' channel were similarly variable. The method used to opsonize bacteria also exerted a considerable influence on the 'high energy' count rate, and indeed, with non-opsonized bacteria the light emission could be recorded in a quite separate energy channel. The particulate stimulator zymosan was especially effective in triggering light emission, as seen by Harvath et al. (1978). The light emission recorded in the 'low energy' part of the energy spectrum remained essentially independent of the nature of the stimulating particle or how it was opsonized. Hatch et al. (1978) found that the chemiluminescence produced by zymosan was less and of shorter duration than that produced by other particulate stimulators, but this was not the case here, nor were there any significant differences in the energy spectra between zymosan-stimulated chemiluminescence and that triggered by opsonized bacteria.

Stevens \& Young (1977) correlated the resistance or sensitivity of $E$. coli strains to phagocytosis with the overall light emission and size of the respiratory burst, and Miller $e$ t al. (1972) did the same with Salmonella and Staphylococcus. The extent of ingestion of the strains used here was not investigated, but Fazeli (1982) observed a correlation between the method used to opsonize bacteria and the extent of ingestion by PMNL. Non-viable bacteria were unable to stimulate PMNL chemiluminescence, the small amount which was seen presumably being a result of a small number of viable cells surviving the killing treatment. Previous reports on this have been contradictory: Stevens \& Young (1977) found an increase in light emission with heat-killed E. coli while DeChatelet $e$ t al. (1974) showed the activity of PMNL metabolic enzymes and phagocytosis to be less when exposed to heat-killed strains rather than live ones. Since the extent of particle entry was not assessed in the present experiments, it is not possible to say whether the killing treatment altered the ability of the bacteria to be recognized by the PMNL or whether some other mechanism was responsible.

At $4{ }^{\circ} \mathrm{C}$, bacterial ingestion was inhibited but attachment was not (Rabinovitch, 1967), and under anaerobiosis, attachment and ingestion occurred but oxidative killing and the respiratory burst were blocked. However, non-oxidative killing mechanisms may still be effective. Under neither of these conditions was 'high energy' light emission detected, although this may have been due to effects on the PMNL metabolism.

In conclusion, multichannel pulse height analysis of phagocytosis-induced chemiluminescence appears to be a promising technique worthy of further investigation. In particular, the reasons for the shape of the spectra obtained, the position of the peak and the nature of the 'shoulder', and the intracellular events with which these are correlated are all unknown at present. 


\section{REFERENCES}

Allen, R. C. (1975a). Halide dependence of the myeloperoxidase-mediated antimicrobial systems of polymorphonuclear leukocytes in the phenomenon of electronic excitation. Biochemical and Biophysical Research Communications 63, 675-683.

Allen, R. C. $(1975 b)$. The role of $\mathrm{pH}$ in the chemiluminescent response of the myeloperoxidasehalide $\mathrm{HOOH}$ antimicrobial system. Biochemical and Biophysical Research Communications 63, 684-691.

ALLEN, R. C. (1977). Evaluation of serum opsonic capacity by quantitating the initial chemiluminescent response from phagocytosing polymorphonuclear leukocytes. Infection and Immunity 15, 828-833.

ALLEN, R. C. (1979). Chemiluminescence from eukaryotic and prokaryotic cells: reducing potential and oxygen requirements. Photochemistry and Photobio$\log y 30,157-163$.

Allen, R. C. \& Loose, L. D. (1976). Phagocytic activation of a luminol-dependent chemiluminescence in rabbit alveolar and peritoneal macrophages. Biochemical and Biophysical Research Communications 69, 245-252.

Allen, R. C., Stjernholm, R. L. \& Steele, R. H. (1972). Evidence for the generation of an elcctronic excitation state(s) in human polymorphonuclear leukocytes and its participation in bactericidal activity. Biochemical and Biophysical Research Communications 47, 679-684.

ANDERSEN, B. R. \& AMIRAULT, H. J. (1979). Important variables in granulocyte chemiluminescence. Proceedings of the Society for Experimental Biology and Medicine 162, 139-145.

ANDERSEN, B. R. \& BRENDZel, A. M. (1978). Use of a unique chemiluminescence spectrometer in a study of factors influencing granulocyte light emission. Journal of Immunological Methods 19, 279-287.

Barbour, A. G., Allered, C. D., Solberg, C. O. \& HILL, H. R. (1980). Chemiluminescence by polymorphonuclear leukocytes from patients with active bacterial infection. Journal of Infectious Diseases 141, 14-26.

DeChatelet, L. R., Mullikin, D., Shirley, P. S. \& MCCall, C. E. (1974). Phagocytosis of live versus heat-killed bacteria by human polymorphonuclear leukocytes. Infection and Immunity 10, 25-29.

Easmon, C. S. F., Cole, P. J., Williams, A. J. \& HASTINGS, M. (1980). The measurement of opsonic and phagocytic function of luminol-dependent chemiluminescence. Immunology 41, 67-74.

FAZELI, S. A. (1982). The ingestion and killing of Escherichia coli by polymorphonuclear leukocytes. $\mathrm{PhD}$ thesis, University of London.

FAZELI, A. \& RichaRDS, L. (1983). The unsuitability of the uridine incorporation assay for the measurement of phagocytosis of Escherichia coli. Journal of General Microbiology 129, 3671-3677.

Grebner, J. V., Mills, E. L., Gray, B. H. \& Quie, P. G. (1977). Comparison of phagocytic and chemiluminescence response of human polymorphonuclear neutrophils. Journal of Laboratory and Clinical Medicine 89, 153-159.

Harvath, L., Amirault, H. J. \& andersen, B. R. (1978). Chemiluminescence of human and canine polymorphonuclear leukocytes in the absence of phagocytosis. Journal of Clinical Investigation 61, $1145-1154$.

HATCh, G. E., Gardner, D. E. \& Menzel, D. B. (1978). Chemiluminescence of phagocytic cells caused by $N$-formylmethionyl peptides. Journal of Experimental Medicine 147, 182-195.

Hemming, V. G., Hall, R. T., Rhodes, P. G., ShigeokA, A. O. \& Hill, H. R. (1976). Assessment of group B streptococcal opsonins in human and rabbit serum by neutrophil chemiluminescence. Journal of Clinical Investigation 58, 1379-1387.

HenRY, J. P. \& Michelson, A. M. (1977). Superoxide and chemiluminescence. In Superoxide and Superoxide Dismutase, pp. 283-290, EMBO Workshop, Banyuls, France. Edited by A. M. Michelson, J. M. McCord \& I. Fridovich. London: Academic Press.

Kossack, R. E., Gubrrant, R. L., Denasen, P., Schadelin, J. \& MANDELl, G. L. (1981). Diminished neutrophil oxidative metabolism after phagocytosis of virulent Salmonella typhi. Infection and Immunity 31, 674-678.

MANDELL, G. L. (1971). Influence of type of ingested particle on human leukocyte metabolism. Proceedings of the Society for Experimental Medicine 137, 1228-1230.

Mason, J. R., SOMerville, H. J. \& PIRT, S. J. (1978). Estimation of biomass within an immobilised cell biocatalyst by means of a luminol assay for haem. Journal of Applied Chemistry and Biotechnology 28, 770-774.

Matthay, M. K., Mentzer, W. C., Wara, D. W., Priesler, H. K., Lameris, N. B. \& AmmanN, A. J. (1981). Evaluation of the opsonic requirements for phagocytosis of Streptococcus pneumoniae serotype VII, XV and XIX by chemiluminescence assay. Infection and Immunity 31, 228-235.

MCCARTHY, J. P., BODROGHY, R. S., JAHRLING, P. B. \& SoBocinski, P. Z. (1980). Differential alterations in host peripheral polymorphonuclear leukocyte chemiluminescence during the course of bacterial and viral infections. Infection and Immunity 30, 824-831.

Miller, R. M., GARBUS, J. \& HoRNICK, R. B. (1972). Lack of enhanced oxygen consumption by polymorphonuclear leukocytes on phagocytosis of virulent Salmonella typhi. Science 175, 1010-1011.

Mills, E. L., RHoll, K. S. \& QuIE, P. G. (1980). Luminol-amplified chemiluminescence: a sensitive method for detecting the carrier state in chronic granulomatous disease. Journal of Clinical Microbiology 12, 52-56.

RABINOVITCH, M. (1967). The dissociation of the attachment and ingestion phase of phagocytosis by macrophages. Experimental Cell Research 46, 19 28.

Rosen, H. \& KLebanofF, S. J. (1976). Chemiluminescence and superoxide production of myeloperoxidase-deficient leukocytes. Journal of Clinical Investigation 58, 50-60.

Stevens, P. \& Young, L. S. (1977). Quantitative granulocyte chemiluminescence in the rapid detection of impaired opsonization of Escherichia coli. Infection and Immunity 16, 796-804.

Van Dyke, K., Trush, M., Wilson, M., Stealey, P.\& MILES, P. (1977). Luminol-dependent chemilumines- 
cence analysis of cellular and humoral defects of phagocytosis using a chem-glo-photometer. Microchemical Journal 22, 463-474.

Williams, A. J. \& Cole, P. J. (1981). The onset of polymorphonuclear leukocyte membrane stimulated metabolic activity. Immunology 43, 733-739.

Williams, A. J., Hastings, M. J. G., Easmon, C. S. F. \& COLE, P. J. (1980). Factors affecting the in vitro assessment of opsonization: a study of the kinetics of opsonization using the technique of phagocytic chemiluminescence. Immunology 41, 903-911.

Wilson, M. E., Trush, M. A., Van Dyke, K., Kyle, J. M., Mullett, M. D. \& Neal, W. A. (1978). Luminol-dependent chemiluminescence analysis of opsonophagocytic dysfunction. Journal of Immunological Methods 23, 315-326. 